\title{
Asynchronous Multi-Centralized Cooperative Localization
}

\author{
Esha D. Nerurkar and Stergios I. Roumeliotis
}

\begin{abstract}
This paper presents a generalized framework for inter-robot information-transfer schemes in Multi-Centralized Cooperative Localization (MC-CL) under asynchronous communication, i.e., when the communication graph associated with the mobile robot network is time-varying and intermittently disconnected. Specifically, two information-transfer schemes, which differ based on their communication bandwidth requirements per link, are discussed. Even under asynchronous communication constraints, these schemes enable robots to compute pose estimates identical to those generated using the centralized CL framework, albeit delayed. For each of these schemes, necessary and sufficient conditions for the communicationgraph connectivity, that enable each robot to generate the centralized estimates, are developed. Moreover, detailed description of these schemes, along with their communication-complexity analysis and analytical results for the expected time delay in obtaining these estimates, are presented. Lastly, simulation results are used to validate the performance (the trade-off between communication link bandwidth and accuracy/delay) of these information-transfer schemes.
\end{abstract}

\section{INTRODUCTION AND RELATED WORK}

Cooperative Localization (CL) is a technique for multirobot pose (i.e., position and orientation) determination. In CL, groups of communicating robots use their relative measurements (e.g., distance, bearing, and orientation) to jointly estimate their poses, resulting in increased accuracy for the entire team [1], [2], [3].

Traditionally in centralized CL, each robot communicates its own measurements to a leader robot or a Fusion Center (FC) that processes these data to generate improved (centralized) pose estimates for the entire team. Depending upon the estimation framework used, various exact centralized algorithms have been proposed. Specifically, an Extended Kalman Filter-based (EKF) algorithm for CL has been introduced in [1], while in [4], the authors present a centralized Maximum Likelihood estimator-based approach to CL. The main drawback of these approaches is that all or most computations are performed centrally, rendering them susceptible to single-point failures of the FC. Moreover, for robot teams navigating in large environments, connectivity constraints (i.e., limited communication range), may prevent robots from sending their measurements to the FC.

An alternative approach that improves the robustness of the system is multi-centralized CL (MC-CL), wherein each robot acts as a $\mathrm{FC}$, i.e., each robot broadcasts its own information to the entire team so that every robot can calculate the centralized pose estimates [5]. Although the

This work was supported by the University of Minnesota (DTC), and the National Science Foundation (EIA-0324864, IIS-0643680, IIS-0811946)

E. D. Nerurkar, and S. I. Roumeliotis are with the Department of Computer Science and Engineering, Univ. of Minnesota USA \{nerurkar, stergios\}@Cs. umn. edu
MC-CL approach of [5] reduces the bandwidth requirements by communicating and processing quantized measurements, it requires a connected communication graph (associated with the mobile robot network) at the time of broadcast, i.e., it requires synchronous communication.

Sub-optimal EKF-based algorithms, that do not require uninterrupted inter-robot communication, are presented in [6], using the Interlaced Kalman filter, and in [7], using stateestimates exchange. An approach based on a hierarchy of EKFs is proposed in [8] where the robot group is divided into sub-teams with leaders estimating the state of their sub-team using an EKF. Furthermore, the leaders themselves can also form sub-teams, resulting into a hierarchical structure. The main drawback of these approaches is that in order to reduce the computational complexity of EKF-based CL, some (or even all in the case of [6]) correlations are ignored, which may lead to overly optimistic and inconsistent estimates.

An approach that maintains these cross-correlations by introducing a bank of EKFs at each robot, is presented in [9]. Each EKF in a bank corresponds to a relative measurement with another robot and accurate book-keeping is used to generate consistent estimates. However, the computational complexity of this approach grows exponentially with the team size. An exact distributed MAP-based algorithm for $\mathrm{CL}$ is presented in [10]. While this algorithm reduces the computational complexity of MAP-based CL, it requires synchronous communication amongst the robots.

Recently, an exact MC-CL approach, that can handle both limited communication range and time-varying communication graphs (asynchronous communication) was proposed in [11]. To achieve this, the authors introduce an informationtransfer scheme wherein each robot broadcasts all its locallyavailable information (its own past and present measurements, as well as past measurements previously received from other robots) to every robot within its communication radius at each time step. The proposed approach is independent of the estimation framework used and enables the robots to obtain delayed centralized estimates. The main drawback of this approach though is its high communication requirement per link, i.e., in communication resourceconstrained applications, there might not exist sufficient bandwidth per link (or time during each exchange) for a robot to communicate all its local information.

The objective of our work is to develop a generalized framework for information-transfer schemes, which differ based on their bandwidth requirements per link, for performing MC-CL under asynchronous communication. Specifically, we present two information-transfer schemes, where each robot communicates: (i) only its own measurements, but for all time steps, and (ii) all measurement information avail- 
able to it, but only from the oldest $q$ time steps. By varying the parameter $q$, a family of information-transfer schemes can be generated, that includes the particular scheme proposed in [11]. Moreover, a trade-off can be achieved between the communication bandwidth requirement per link (increases with $q$ ) and the time delay in obtaining the centralized estimates (decreases with increasing $q$ ). By choosing an appropriate information-transfer scheme, based upon the communication resources available to the team, each robot can generate pose estimates identical to the centralized estimates (no approximations), but delayed. The proposed informationtransfer schemes are independent of the estimator used, while the computational complexity per robot is identical to the corresponding centralized algorithm for CL.

In what follows, we first describe the problem formulation and each of the data-transfer schemes in detail along with their communication-complexity analysis. Then, for each of the proposed schemes, we develop necessary and sufficient conditions for communication-graph connectivity which, if satisfied, guarantee that each robot will be able to generate the centralized pose estimates, albeit delayed. Furthermore, we present analytical results for the expected time delay in obtaining the centralized estimates for some of these schemes. Lastly, we present simulation results that compare the performance (trade-off between communication bandwidth per link and accuracy/delay) of the proposed information-transfer schemes.

\section{Problem Formulation}

Consider a team of $N$ communicating robots navigating in $2 \mathrm{D}$ while performing CL. The state vector $\mathbf{x}_{k}=$ $\left[\mathbf{x}_{k}^{1}, \mathbf{x}_{k}^{2^{T}}, \ldots, \mathbf{x}_{k}^{N^{T}}\right]^{T}$, where $\mathbf{x}_{k}^{i}=\left[x_{k}^{i}, y_{k}^{i}, \phi_{k}^{i}\right]^{T}, i=$ $1, \ldots, N$, contains the position and orientation of all robots at time-step $k$. Note that the team can use any estimation algorithm of its choice (e.g., EKF, MAP estimator, Particle filters, etc.) for pose determination. Each robot is equipped with proprioceptive (odometry) sensors that provide linear, $v_{m_{k}}^{i}$, and rotational, $\omega_{m_{k}}^{i}$, velocity measurements. The motion model for robot $i$ is given by [10]:

$$
\mathbf{x}_{k}^{i}=\mathbf{f}\left(\mathbf{x}_{k-1}^{i}, \mathbf{u}_{k-1}^{i}, \mathbf{w}_{k-1}^{i}\right), \quad \mathbf{w}_{k-1}^{i} \sim \mathcal{N}\left(\mathbf{0}, \mathbf{Q}_{k-1}^{i}\right),
$$

where $\mathbf{f}$ is a general non-linear function, $\mathbf{u}_{k-1}^{i}=$ $\left[v_{m_{k-1}}^{i}, \omega_{m_{k-1}}^{i}\right]^{T}$ is the control input and $\mathbf{w}_{k-1}^{i}=$ $\left[w_{v_{k-1}}^{i}, w_{\omega_{k-1}}^{i}\right]^{T}$ is the process noise.

Additionally, all robots have exteroceptive sensors that allow them to uniquely identify other robots in the team and measure their relative distance and bearing. The measurement model for robot $i$ measuring robot $j$ is:

$$
\mathbf{z}_{k}^{i, j}=\mathbf{h}\left(\mathbf{x}_{k}^{i}, \mathbf{x}_{k}^{j}\right)+\mathbf{n}_{k}^{i, j}, \quad \mathbf{n}_{k}^{i, j} \sim \mathcal{N}\left(\mathbf{0}, \mathbf{R}_{k}^{i, j}\right),
$$

with $\mathbf{h}=\left[d_{k}^{i, j}, \theta_{k}^{i, j}\right]^{T}$, where $d_{k}^{i, j}, \theta_{k}^{i, j}$ are the true distance, bearing respectively, from robot $i$ to robot $j$ at time-step $k$ and $\mathbf{n}_{k}^{i, j}=\left[n_{d_{k}}^{i, j}, n_{\theta_{k}}^{i, j}\right]^{T}$ is the measurement noise.

Let $R=\{1, \ldots, N\}$ denote the set of indices of all robots in the team and let $M_{k}^{i}$ denote the set of measurements generated by robot $i$ at time-step $k$, i.e.,

$$
M_{k}^{i}=\left\{\mathbf{u}_{k}^{i}, \mathbf{z}_{k}^{i, j}\right\}, \forall j \in R, j \neq i, d_{k}^{i, j} \leq d_{\text {max }}^{i},
$$

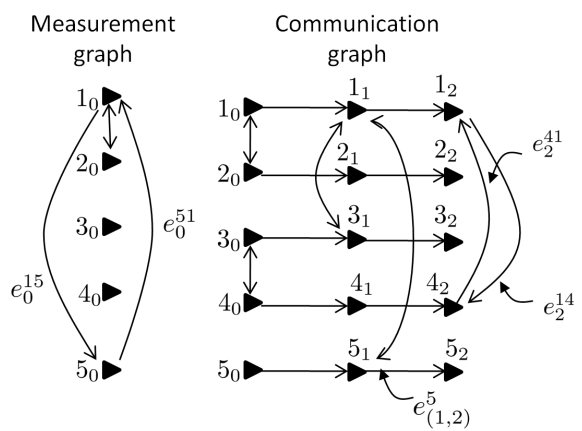

Fig. 1: (Left) Measurement graph for a team of 5 robots at timestep 0. (Right) Communication graph for a team of 5 robots from time-steps 0 to 2 .

where $d_{\max }^{i}$ is the maximum sensing radius of robot $i$. At time-step $k$, robot $i$ can communicate with robot $j$ only if $d_{k}^{i, j} \leq r^{i}$, where $r^{i}$ is the communication radius of robot $i$. Let $C_{k}^{i}$ denote the set of indices of all robots that lie within the communication radius of robot $i$ at time-step $k$. Note that we assume bidirectional communication among the robots.

Depending on the robots' motion, each robot may sense and communicate with a different sub-team at every time step, i.e., both the measurement graph and the communication graph, associated with the mobile robot network, can be time-varying and incomplete. Specifically, the measurement graph for the robot team, at time-step $k$, is a directed graph where node $i_{k}$ denotes robot $i$ at time-step $k$ and edge $e_{k}^{i j}$ exists if robot $i$ obtains a relative measurement to robot $j$ (cf. Fig. 1). The communication graph considers a timewindow from time-step $k$ to $k+p$, and represents the flow of information in the robot network. Here, edge $e_{m}^{i j}$, $m \in\{k, \ldots, k+p\}$ exists, if robot $i$ can communicate information to robot $j$ at time-step $m$. Also, edge $e_{(m, m+1)}^{i}$ always exists, indicating that robot $i$ 's information from timestep $m$ is always available to itself at time-step $m+1$.

We denote by $T_{k}^{i-}$ the set of all information that is locallyavailable to robot $i$ at time-step $k$, before it communicates with any other robot in the team. The set $T_{k}^{i+}$ denotes the information that is locally-available to robot $i$ after it has received information from all robots within communication range, i.e.,

$$
T_{k}^{i+}=T_{k}^{i^{-}} \cup\left(\bigcup_{j \in C_{k}^{i}} S_{k}^{j}\right)
$$

where $S_{k}^{j}$ denotes the information that is communicated by robot $j$ to robot $i$ at time-step $k$. The contents of $S_{k}^{j}$ depend upon the information-transfer scheme used. Furthermore, $T_{k}^{i-}$ can be expressed as:

$$
T_{k}^{i^{-}}=T_{(k-1)}^{i^{+}} \cup M_{k}^{i}
$$

As the robot team moves around in the environment, depending on the evolution of the communication graph and the information-transfer scheme used, each robot starts accumulating information about the entire team. Let us assume that at time-step $k+m$, where $m \geq 0$, robot $i$ obtains all information about the team up to time-step $k$, 
Time-step 1, Robot 1 Time-step 2, Robot 1
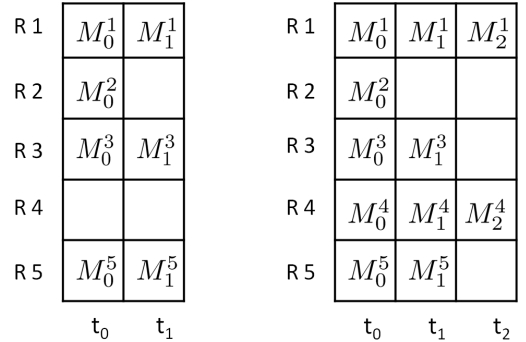

Fig. 2: Information tables for robot 1 at time-steps 1 (left) and 2 (right). $t_{k}$ : time-step $k, R i$ : Robot $i$.

i.e., $T_{k+m}^{i^{+}} \supseteq\left(\bigcup_{j \in R} M_{0: k}^{j}\right)$. Using this information, robot $i$ can calculate pose estimates ${ }^{1}$, denoted by $\hat{\mathbf{x}}_{k \mid k}$, for the entire team, that are identical to those generated if using centralized CL. But since these estimates for time-step $k$ can be calculated no earlier than time-step $k+m$ (due to the delay in information transfer), we denote these delayed estimates by $\hat{\mathbf{x}}_{k \mid k}^{k+m}$.

Consider the example shown in Fig. 1. The information tables in Fig. 2 depict the information that is available to robot 1 at time-steps 1 and 2 . Note that robot 1 does not have any information about robot 4 at time-step 1 . Now at time-step 2, assume that robot 4 communicates $S_{2}^{4}=M_{0: 2}^{4}$ to robot 1 . Thus at time-step 2, robot 1 acquires all information about the team up to time-step 0 and can hence calculate the centralized pose estimates ${ }^{2} \hat{\mathbf{x}}_{0 \mid 0}^{2}$.

Thus the flow of information between robots is governed not only by the communication graph, but also by the set $S_{k}^{j}$, which is determined by the information-transfer scheme used by the robot team. The choice of an appropriate informationtransfer scheme, in turn is influenced by the communication bandwidth per link available to the robot team.

Before proceeding to the next section that presents our proposed information-transfer schemes, we briefly recap the notation used in this paper:

- $R$ : set of indices of all robots in the team.

- $C_{k}^{i}$ : set of indices of robots that can communicate with robot $i$ at time-step $k$.

- $M_{k}^{i}$ : set of proprioceptive and exteroceptive measurements generated by robot $i$ at time-step $k$.

- $T_{k}^{i-} / T_{k}^{i+}$ : set of all information that is available to robot $i$ up to and including time-step $k$, before/after communication with neighboring robots.

- $S_{k}^{i}$ : set of information communicated by robot $i$ to all robots within its communication radius at time-step $k$.

\section{INFORMATION TRANSFER SCHEMES}

\section{A. Scheme 1: Own Information Transfer only}

1) Description: In this scheme, each robot communicates only its own proprioceptive and exteroceptive measurement

\footnotetext{
${ }^{1} \hat{\mathbf{x}}_{l \mid m}$ denotes pose estimates at time-step $l$, using all measurement information up to time-step $m$.

${ }^{2}$ Note that in addition to the delayed centralized estimate, each robot can compute a causal estimate for its pose given all measurements currently available to it.
}

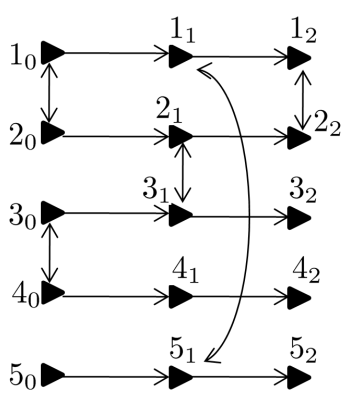

(A)

Fig. 3: Scheme 1. Communication graph for a team of 5 robots from time-steps 0 to 2 .

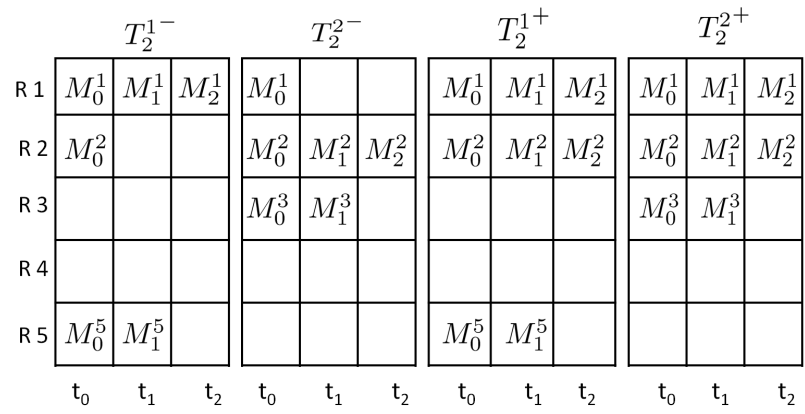

Fig. 4: Scheme 1. Information tables for robots 1 and 2 at timestep 2, before and after communication.

information to other robots. Assume that: (i) robot $i$ and robot $j$ last communicated with each other at time-step $k$, and (ii) the next communication opportunity for these two robots arises at time-step $k+p, p \geq 1$. Using Scheme 1 , robot $\ell, \ell \in$ $\{i, j\}$, will communicate the set $S_{(k+p)}^{\ell}=M_{k+1: k+p}^{\ell}$ at timestep $k+p$. Therefore, after communication, the information set $T_{(k+p)}^{i^{+}}$for each robot $i, i \in R$ will contain:

$$
T_{(k+p)}^{i^{+}}=T_{(k+p)}^{i^{-}} \cup\left(\bigcup_{j \in C_{(k+p)}^{i}} S_{(k+p)}^{j}\right) .
$$

Consider the example shown in Figs. 3 - 4. Since robots 1 and 2 last communicated at time-step 0 , when they now meet at time-step 2, robot 1 will communicate only its own information from time-steps 1 and 2, i.e., $S_{2}^{1}=M_{1: 2}^{1}$, to robot 2. Similarly, robot 2 will also communicate only its own information from time-steps 1 and 2, i.e., $S_{2}^{2}=M_{1: 2}^{2}$, to robot 1. As a result, both robots will have identical information for each other but not for the rest of the team.

Note that in the information-transfer scheme proposed in [11], each robot communicates all its available information, i.e., information from all rows in its information table. By contrast, in Scheme 1 each robot communicates information only from a single row (corresponding to itself) in its information table.

2) Communication complexity analysis: When the two robots communicate after $p$ time steps, each robot $i$ has to communicate: (i) its $p$ proprioceptive measurements, $\mathbf{u}_{k+1: k+p}^{i}$, and (ii) its exteroceptive measurements, $\mathbf{z}_{K}^{i, \ell}, \forall \ell \in R, \ell \neq i, d_{K}^{i, \ell} \leq d_{\max }^{i}, K=k+1, \ldots, k+p$. Therefore, the communication cost/amount of information 
that has to be transferred over the communication link is $O\left(\left|\mathbf{u}_{k+1: k+p}^{i}\right|+\sum_{K=k+1}^{k+p}\left|\mathbf{z}_{K}^{i, \ell}\right|\right)$, where $|g|$ denotes the cardinality of $g$. Here, the first term depends only on $p$ which is determined by the frequency of inter-robot communication, while $\left|\mathbf{z}_{K}^{i, \ell}\right|$ also depends on the number of robots that are sensed by robot $i$ per time step, i.e., the outdegree of robot $i$ in the measurement graph. Therefore, for a particular application, if the frequency of inter-robot communication and the average outdegree of the measurement graph can be approximated before-hand, communication bandwidth per link can be reserved accordingly for CL. Moreover, this information-transfer scheme is well suited for applications where the robots communicate often ( $p$ remains small).

3) Communication graph connectivity analysis: We now present the necessary and sufficient conditions (on communication-graph connectivity), that if satisfied, guarantee that each robot in the team can compute the centralized pose estimates.

Lemma 1: Robot $i$ can compute $\hat{\mathbf{x}}_{k \mid k}^{\max \left\{k_{j}\right\}}$ if and only if robot $j, \forall j \in R, j \neq i$, communicates with robot $i$ at time-step $k_{j}$, where $k_{j} \geq k$.

Proof: Details of this lemma can be found in [12].

Lastly, robot $i$ can discard all information (own and other robots' measurements) up to time-step $k$ at time-step $\max \left\{k_{j}\right\}$, i.e., after calculating $\hat{\mathbf{x}}_{k \mid k}^{\max \left\{k_{j}\right\}}$. Since we assume bidirectional communication between robots, if robot $i$ has received information, up to time-step $k$ from all other robots, this implies that robot $i$ has communicated its own information about time-step $k$ to all other robots too. Hence it can safely discard all the information and retain only the corresponding centralized estimates.

4) Expected time delay analysis: Let $p_{m}^{j i}$ be the probability that edge $e_{m}^{j i}$ exists in the communication graph. For simplicity, we assume that: (i) this probability remains constant over time (denoted by $p^{j i}$ ), and (ii) $p^{j i}=p^{i j}$. Therefore, the probability that edge $e_{m}^{j i}$ does not exist is $\left(1-p^{j i}\right)$. The expected time delay, $E_{i}$ (time delay), for robot $i, \forall i \in R$ is given by:

$$
E_{i}(\text { time delay })=\sum_{t=0}^{\infty} t \times p_{i}(\text { time delay }=t),
$$

where $p_{i}$ (time delay $=t$ ) is the probability that the centralized estimates for time-step $k$ can be calculated no earlier than time-step $k+t$.

Consider the event where the time delay in obtaining the centralized estimates is less than or equal to $t$, i.e., time delay $\leq t$. For this event to occur, robot $j$, $\forall j \in R, j \neq i$, should communicate with robot $i$ at least once up to time-step $k+t$, i.e., at least one edge $e_{m}^{j i}$, $k \leq m \leq k+t$ should exist in the communication graph for every robot $j$. The probability that there exists at least one edge between robot $j$ and robot $i$ from time-step $k$ to time-step $k+t$ is given by:

$$
p_{i}\left(\exists e_{m}^{j i}, k \leq m \leq k+t\right)=1-\left(1-p^{j i}\right)^{t+1} .
$$

\footnotetext{
${ }^{3}$ Note that $\hat{\mathbf{x}}_{k \mid k}^{m}$ indicates that the centralized estimates for time-step $k$ can be calculated no earlier than time-step $m$.
}

Therefore the probability that there exists at least one edge between robot $i$ and every other robot in the team from timestep $k$ to $k+t$, i.e., $p_{i}($ time delay $\leq t)$ is given by:

$p_{i}\left(\exists e_{m_{j}}^{j i}, k \leq m_{j} \leq k+t, \forall j \in R, j \neq i\right)=\prod_{\substack{j=1 \\ j \neq i}}^{N}\left(1-\left(1-p^{j i}\right)^{t+1}\right)$.

Employing an analogous expression for $p_{i}($ time delay $\leq(t-1))$, we have:

$$
\begin{aligned}
& p_{i}(\text { time delay }=t) \\
& =p_{i}(\text { time delay } \leq t)-p_{i}(\text { time delay } \leq(t-1)) \\
& =\prod_{\substack{j=1 \\
j \neq i}}^{N}\left(1-\left(1-p^{j i}\right)^{t+1}\right)-\prod_{\substack{j=1 \\
j \neq i}}^{N}\left(1-\left(1-p^{j i}\right)^{t}\right) .
\end{aligned}
$$

Substituting (10) in (7) yields:

$$
\begin{aligned}
& E_{i} \text { (time delay) } \\
& =\sum_{t=0}^{\infty} t \times\left(\prod_{\substack{j=1 \\
j \neq i}}^{N}\left(1-\left(1-p^{j i}\right)^{t+1}\right)-\prod_{\substack{j=1 \\
j \neq i}}^{N}\left(1-\left(1-p^{j i}\right)^{t}\right)\right) .
\end{aligned}
$$

Thus, by modeling $p^{j i}$ based on the network topology over time, the above formula can be used to accurately model the expected time delay ${ }^{4}$.

\section{B. Scheme 2: Information Transfer from q oldest time steps}

Recall that in Scheme 1, information is transferred rowwise, i.e., each robot communicates the entire row corresponding to itself in its information table (cf. Fig. 4). Note, however that, for small robot teams communicating infrequently, the amount of information available in each row is usually significantly larger compared to the data stored in each column. In such cases, and in order to reduce the time delay in obtaining centralized estimates, information should be communicated column-wise, i.e., each robot should communicate all its locally available information, starting with the oldest time step first (i.e., the first column of its information table). The information-transfer scheme that we now propose is based on this general framework, where the parameter $q$ defines the number of columns (time steps) that are communicated. When $q=1$, all information from only the oldest time step is broadcast to neighbors. As $q$ increases, information from more time steps is communicated, thus increasing the communication requirement per link, but reducing the delay in obtaining centralized estimates. Finally, the case where $q=\infty$ corresponds to the informationtransfer scheme of [11], where each robot transfers all its locally-available information ${ }^{5}$. We now present details for the two extreme cases, $q=1$ and $q=\infty$, while details for the general case can be found in [12].

\footnotetext{
${ }^{4}$ Recursive formulae for the expected time delay, both for the number of robots and time steps, are presented in [12].

${ }^{5}$ We use the notation $q=\infty$ to indicate that information about an arbitrarily large number of time steps might have to be communicated, depending upon the evolution of the communication graph. For example, if two robots, that have not communicated for a long time period meet, then $q$ can take on a very large value.
} 


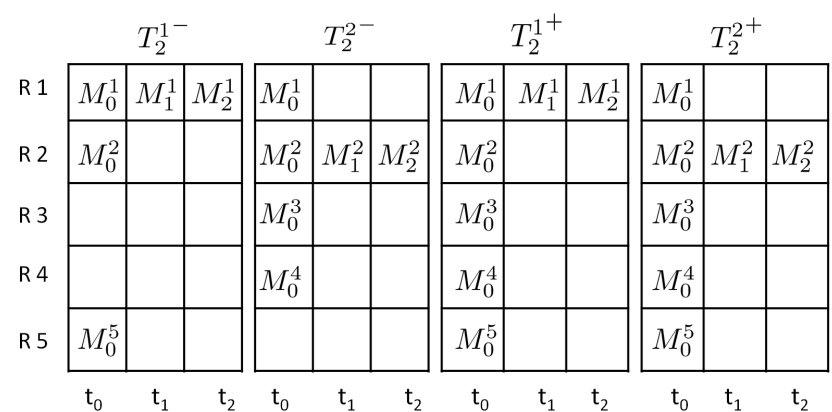

Fig. 5: Scheme 2 with $q=1$. Information tables for robots 1 and 2 at time-step 2 , before and after communication.

1) Description: We first consider the case when $q=1$. Here, each robot communicates all its locally-available measurement information from the oldest time step only. Assume that robot $i$ has the oldest information about time-step $k$, for itself, i.e., $M_{k}^{i}$, and for a subset, $R_{i}$, of the other robots in the team, i.e., $\left(\bigcup_{\ell \in R_{i}} M_{k}^{\ell}\right)$. If robot $i$ communicates with robot $j$ at time-step $k_{j} \geq k$, robot $i$ will transfer $S_{k_{j}}^{i}=M_{k}^{i} \cup\left(\bigcup_{\ell \in R_{i}} M_{k}^{\ell}\right)$. Thus, after communication the information set for robot $j$ at time-step $k_{j}$ will contain:

$$
T_{k_{j}}^{j+}=T_{k_{j}}^{j-} \cup\left(\bigcup_{r \in C_{k_{j}}^{j}} S_{k_{j}}^{r}\right)
$$

For the communication graph in Fig. 3, Fig. 5 shows the information tables for $T_{2}^{1^{-}}$and $T_{2}^{2-}$, obtained using Scheme 2 with $q=1$. When robots 1 and 2 communicate at time-step 2, robot 1 communicates $S_{2}^{1}=M_{0}^{5}$ to robot 2 (it need not communicate robot 2's data to robot 2 itself), while robot 2 communicates $S_{2}^{2}=M_{0}^{3} \cup M_{0}^{4}$ to robot 1 . Now since both robots have all information up to time-step 0 , they can each calculate $\hat{\mathbf{x}}_{0 \mid 0}^{2}$. Finally, we note that the oldest information available to both robots after communication is the union of their individual oldest information before communication.

We now consider the case when $q=\infty$. While this scheme was originally proposed in [11], in this section we present additional communication complexity and expected time delay analysis. Furthermore, we also discuss a book-keeping technique that can be used by the robots to reduce communication overhead. In this scheme, each robot communicates all its locally-available measurement information, i.e., its own information and also the information that it received from other robots in the team. Assuming that robots $i$ and $j$ last communicated at time-step $k$, when they next communicate at time-step $k+p, p \geq 1$, each robot $\ell, \ell \in\{i, j\}$, will communicate the set $S_{(k+p)}^{\ell}$ where:

$$
S_{(k+p)}^{\ell}=M_{k+1: k+p}^{\ell} \cup\left(\bigcup_{k_{r}=k}^{k+p-1}\left(\bigcup_{r \in C_{k_{r}}^{\ell}} S_{k_{r}}^{r}\right)\right),
$$

where $\left(\bigcup_{k_{r}=k}^{k+p-1}\left(\bigcup_{r \in C_{k_{r}}^{\ell}} S_{k_{r}}^{r}\right)\right)$ represents all the information that robot $\ell$ received from other robots, from time-step $k$ up to time-step $k+p-1$. Therefore, after communication, the

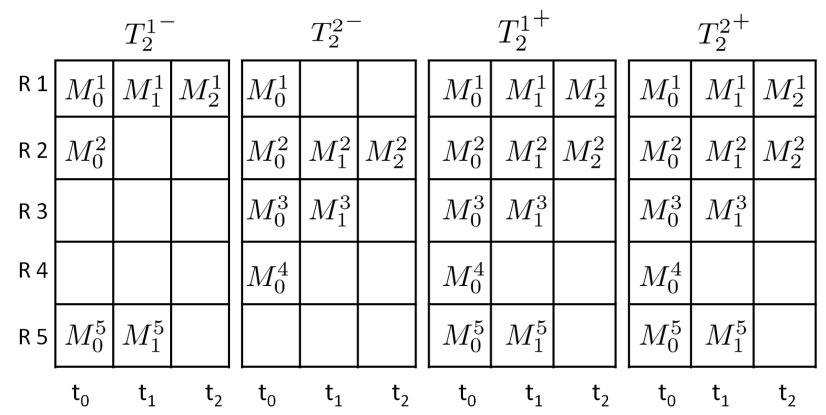

Fig. 6: Scheme 2 with $q=\infty$. Information tables for robots 1 and 2 at time-step 2 , before and after communication.

information set $T_{(k+p)}^{i^{+}}$for each robot $i, i \in R$ will contain:

$$
T_{(k+p)}^{i^{+}}=T_{(k+p)}^{i^{-}} \cup\left(\bigcup_{j \in C_{(k+p)}^{i}} S_{(k+p)}^{j}\right) .
$$

Using Scheme 2 with $q=\infty$, for the same communication graph as in Fig. 3, generates $T_{2}^{1^{-}}$and $T_{2}^{2^{-}}$as shown in Fig. 6. Now when robots 1 and 2 communicate at time-step 2, robot 1 communicates $S_{2}^{1}=M_{1: 2}^{1} \cup M_{0: 1}^{5}$ to robot 2, while robot 2 communicates $S_{2}^{2}=M_{1: 2}^{2} \cup M_{0: 1}^{3} \cup M_{0}^{4}$ to robot 1 . Similar to Scheme 2 with $q=1$, both robots will be able to calculate $\hat{\mathbf{x}}_{0 \mid 0}^{2}$, but note that the information available about future time steps is different in both schemes. In particular, contrary to the case of $q=1$, for $q=\infty, T_{2}^{1^{+}}=T_{2}^{2^{+}}=$ $T_{2}^{1^{-}} \cup T_{2}^{2^{-}}$.

Since each robot communicates information about other robots too, depending on the evolution of the communication graph, there might exist an overlap between the information that is locally-available to two communicating robots. Thus by using an efficient book-keeping routine, the communication of redundant information can be avoided. In our proposed book-keeping approach, robot $i$ first communicates a list of tuples $L_{i}=\left\{\left(\ell, k_{\ell}^{i}\right)\right\}$, where $\ell$ is the id of the robot whose measurement information is available to robot $i$, and $k_{\ell}^{i}$ is the latest time step for which this information is available. Once robot $j$ receives this list from robot $i$, robot $j$ calculates the set of robot ids, $V_{j}=\{v \mid(v \in$ $\left.\left.L_{i}, v \in L_{j}, k_{v}^{j} \geq k_{v}^{i}\right) \vee\left(v \in L_{j}, v \notin L_{i}\right)\right\}$, of robots whose information has to be communicated to robot $i$ and communicates only:

D1: $\left\{\mathbf{u}_{K}^{v}, \mathbf{z}_{K}^{v, r}\right\}$, where $v \in L_{j}, v \in L_{i}, \forall r \in R, r \neq$ $v, d_{K}^{v, r} \leq d_{\max }^{v}$ where $K=k_{v}^{i}+1, \ldots, k_{v}^{j}$.

D2: $\left\{\mathbf{u}_{K}^{v}, \mathbf{z}_{K}^{v, r}\right\}$, where $v \in L_{j}, v \notin L_{i}, \forall r \in R, r \neq$ $v, d_{K}^{v, r} \leq d_{\max }^{v}$ where $K=m, \ldots, k_{v}^{j}$ and $m$ is the earliest time step for which data about robot $v$ is available.

2) Communication complexity analysis: When $q=1$, since each robot communicates only its oldest-available information, when robots $i$ and $j$ communicate, the communication cost/amount of information that has to be transferred per communication link is $O\left(\left|M_{k}^{i}\right|+\sum_{\ell \in R_{i}}\left|M_{k}^{\ell}\right|\right)=$ $O\left(\left|\mathbf{u}_{k}^{i}\right|+\left|\mathbf{z}_{k}^{i, p}\right|+\sum_{\ell \in R_{i}}\left(\left|\mathbf{u}_{k}^{\ell}\right|+\left|\mathbf{z}_{k}^{\ell, s}\right|\right)\right.$, where $p$ and $s$ denote the robots that were observed by robots $i$ and $\ell$ respectively, 
at time-step $k$. Importantly for this approach, the communication cost depends upon the outdegree of the measurement graph and the number of robots in the team, but it is independent of the frequency of inter-robot communication, i.e., irrespective of the time that has elapsed since robots $i$ and $j$ last communicated, when robot $i$ next communicates with robot $j$, information for only a single time step will be communicated. This makes this scheme suitable for use in severe communication-resource constrained applications, but delays the calculation of centralized estimates.

For $q=\infty$, when robots $i$ and $j$ communicate after $p$ time steps, robot $i$ first communicates its list $L_{i}$ and then depending on the list $L_{j}$ that it obtains from robot $j$, it communicates the non-redundant measurement information corresponding to $\left\{L_{i} \backslash L_{j}\right\}$. Therefore, the total information that has to be communicated by robot $i$ to robot $j$ when they meet after $p$ time steps is $\left|L_{i}\right|$ plus all the information mentioned in the previous section (sets $D 1$ and $D 2$ listed in book-keeping). While this scheme has the highest communication complexity per link, as compared to Scheme 1 and Scheme 2 with $q=1$, it has the lowest time delay in obtaining the centralized estimates. Therefore, this scheme is applicable to scenarios where there is no constraint on the available communication bandwidth.

3) Communication graph connectivity analysis: We now present the necessary and sufficient condition for obtaining centralized estimates for both these cases.

Lemma 2: Robot $i$ can compute $\hat{\mathbf{x}}_{k \mid k}^{\max \left\{k_{j}\right\}}$ if and only if there exists $j_{k} \rightarrow i_{k_{j}}, \forall j \in R, j \neq i$, where $k_{j} \geq k$. The notation $j_{k} \rightarrow i_{k_{j}}$ denotes a path in the communication graph from node $j_{k}$ to node $i_{k_{j}}$, such that information about robot $j$ up to time-step $k$, i.e., $M_{0: k}^{j}$, is available to robot $i$, no earlier than at time-step $k_{j} \geq k$.

Proof: Derivation is shown in [12] $]^{6}$.

While the statement of the above theorem is identical for both cases (i.e., they both require the existence of an appropriate path in the communication graph), we now highlight the difference in these paths for the two cases. Let us assume that robots $i$ and $j$ communicate at time-step $k$. When $q=\infty$, each robot transfers all its locally-available information, and therefore the information communicated by robot $i$, at time-step $k$, will include $M_{k}^{i}$. But when $q=1$, since only the oldest information is transmitted, the information communicated by robot $i$ will include $M_{k}^{i}$, only if $k$ is the oldest time step for which measurement information is available to robot $i$. For example, in Fig. 6, for $q=\infty$, when robots 1 and 2 communicate at timestep 2, robot 1 communicates information about time-step 2 too. On the other hand for $q=1$ (cf. Fig. 5), at time-step 2 robot 1 communicates information only about the oldest time step, i.e., time-step 0 . Robot 1 will communicate information about time-step 2 only after information about time-steps 0 and 1 has been discarded. Thus even though both cases require the existence of a path in the communication graph between robot $j$ at time-step $k$ and robot $i$ at time-step $k_{j}$, these communication paths may not be the same.

\footnotetext{
${ }^{6}$ The derivation for Scheme 2 with $q=\infty$ has been presented in [11].
}

Regardless of the choice of $q$, in Scheme 2 robot $i$ can discard all information about time-step $k$, only after: (i) it has computed the centralized estimates for time-step $k$, and (ii) it has ensured that these centralized estimates (in the form of raw measurements) have been communicated to all other robots in the team. Robot $i$ can ensure this by communicating this information to all robots personally, or by communicating with another robot in the team that has satisfied conditions (i) and (ii) above, whichever happens earlier ${ }^{7}$.

4) Expected time delay analysis: In Scheme 1, since each robot communicates only its own information, $E_{i}$ (time delay), $\forall i \in R$, depends only upon the $(N-1)$ possible communication links per time step, from other robots in the team to robot $i$. Thus the edges $e_{k}^{\ell r}, \forall \ell, r \in R \backslash i$, for any time-step $k$, do not affect $E_{i}$ (time delay). By contrast, in Scheme 2, since each robot can communicate information about all robots, $E_{i}$ (time delay) can be affected by any of the $N(N-1)$ possible edges per time step, depending upon the evolution of the communication graph. We now present the expected time delay analysis for a team of 3 robots using Scheme 2 with $q=\infty$. Our future work includes generalization of this result to the case of $N$ robots.

Assume that we want to calculate the centralized estimates for time-step $k$. Let $I_{t}^{i}=p_{i}($ time delay $>t)$ be the probability that the time delay in computing these centralized estimates is greater than $t$ for robot $i$. Thus, $p_{i}($ time delay $\leq t)=1-I_{t}^{i}$ and $p_{i}($ time delay $=t)=$ $-I_{t}^{i}+I_{t-1}^{i}$. We assume $p^{i j}=p, \forall i, j \in R$. Now in order to obtain an expression for $E_{i}$ (time delay) (cf. (7)), we need to calculate $I_{t}^{i}$. The event (time delay $>t$ ) can be expressed as the union of two mutually-exclusive sub-events, (E1) and (E2), which in turn are expressed as the intersection of two independent sub-events each:

E1: (E1a) Neither of the other two robots in the team communicate with robot $i$ at time-step $k+t$, i.e., edges $e_{k+t}^{j i}, \forall j \in R, j \neq i$, do not exist, and (E1b) the event (time delay $>t-1$ ) is true for robot $i$. The probability of event $(\mathrm{E} 1 \mathrm{a})$ is $(1-p)^{2}$. Thus probability of event (E1) is given by $(1-p)^{2} p_{i}$ (time delay $\left.>t-1\right)$.

E2: (E2a) Either of the other two robots in the team (say robot $j$ ) communicates with robot $i$ at time-step $k+t$, i.e., the edge $e_{k+t}^{j i}$ exists at time-step $k+t$, and (E2b) the third robot in the team (say robot $\ell$ ) does not communicate with either robot $i$ or robot $j$ from timestep $k$ to time-step $k+t-1$, i.e., edges $e_{m}^{\ell i}, \ell \in R$, $\ell \neq i, j$, for $k \leq m \leq k+t-1$ do not exist. Now the probability of event (E2a) is given by $2(1-p) p$, while the probability of event $(\mathrm{E} 2 \mathrm{~b})$ is given by $(1-p)^{2 t}$. Therefore the probability of event (E2) is given by $2 p(1-p)^{2 t+1}$.

From the union of (E1) and (E2) we have:

\footnotetext{
${ }^{7}$ In the scheme proposed in [11], robot $i$ discards the raw measurements at time-step $k$, as soon as it has calculated the centralized estimate for this time step and then communicates this centralized estimate to other robots. Note that irrespective of whether the raw measurements themselves or the centralized estimate computed from these measurements are communicated, the information content remains the same.
} 
TABLE I: Expected Time Delay Analysis

$$
I_{t}^{i}=p_{i}(\text { time delay }>t-1)(1-p)^{2}+2 p(1-p)^{2 t+1} .
$$

Note that $p_{i}$ (time delay $\left.>0\right)=\left(1-p^{2}\right)$ and $p_{i}($ time delay $=0)=p^{2}$. Substituting (15) in (7) yields $E_{i}($ time delay) as:

$$
\begin{aligned}
& \sum_{t=1}^{\infty} t \times\left(I_{t-1}^{i}-I_{t}^{i}\right) \\
& =\sum_{t=1}^{\infty} t \times\left(p_{i}(\text { time delay }>t-1)\left(2 p-p^{2}\right)-2 p(1-p)^{2 t+1}\right) .
\end{aligned}
$$

\section{Simulations}

In this section, we compare the performances of the three information-transfer schemes discussed in this paper. The objective of our simulation studies is twofold: (i) to compare the expected time delay in obtaining the centralized estimates for these schemes, and (ii) to study the trade-off between the accuracy of pose estimates for the robot team at time-step $k$, obtained using information available at time-step $k$ (without including the delayed information), and the communication bandwidth requirements per link for these schemes.

To study the behavior of the expected time delay for these schemes, we carried out Monte-Carlo runs on a general mobile robot network of $N$ robots over 1000 time steps, with $p^{i j}=0.5, \forall i, j \in R$, for each scheme. Furthermore, by varying $N$, we studied the effect of the size of the robot team on the delay in obtaining the centralized estimates. These results are presented in Table I. Firstly, we note that for Scheme 1 and Scheme $2(q=\infty)$ for $N=3$, the simulation results for the expected time delay corroborate our analytical results. Next, from Table I we see that the expected time delay is lowest for Scheme $2(q=\infty)$, irrespective of the size of the robot team. This result is expected, because as compared to the other two approaches, this scheme has the highest rate of information transfer. However, this improvement in performance is comes at the cost of increased communication complexity (cf. Sec. IIIB.2).

More importantly, in Scheme $2(q=\infty)$, as the number of robots increases, the expected time delay goes on decreasing and finally becomes 1 time step. To corroborate this result, consider the $p_{i}$ (time delay $\left.>1\right)$ for Scheme $2(q=\infty)$ for a team of $N$ robots [12]:

$$
\begin{aligned}
& p_{i}(\text { time delay }>1) \\
& =\sum_{r=1}^{N-1}\left(\begin{array}{c}
N-1 \\
r
\end{array}\right)(1-p)^{r} p^{(N-1-r)}\left(1-\left(1-(1-p)^{(N-r)}\right)^{r}\right) .
\end{aligned}
$$

Furthermore, $p_{i}($ time delay $=1)$ can be expressed as:

$$
\begin{aligned}
& p_{i}(\text { time delay } \leq 1)-p_{i}(\text { time delay }=0) \\
& =1-p_{i}(\text { time delay }>1)-p^{N-1} .
\end{aligned}
$$

Table II shows the values of $p_{i}($ time delay $=1)$ for different team sizes. From the table we see that as $N$ increases, $p_{i}$ (time delay $=1$ ) goes very quickly to 1 .

\begin{tabular}{|c||c|c|c|}
\hline Team size & Sch. 1 (theor./sim.) & Sch. 2, $q=\infty$ & Sch. 2, $q=1$ \\
\hline 3 & $1.6667 / 1.6488$ & $1.2333 / 1.2203$ & 66.0237 \\
\hline 5 & $2.5048 / 2.4867$ & 1.2876 & 66.7684 \\
\hline 10 & $3.5813 / 3.5495$ & 1.1673 & 69.9089 \\
\hline 20 & $4.6183 / 4.5356$ & 1.0231 & 70.6912 \\
\hline 50 & $5.9621 / 5.8811$ & 1 & 72.5475 \\
\hline 100 & $6.9694 / 7.0152$ & 1 & 74.2389 \\
\hline
\end{tabular}

TABLE II: $p_{i}($ time delay $=1)$ for Scheme $2(q=\infty)$

\begin{tabular}{|c|c|}
\hline Team size & $p_{i}($ time delay $=1)$ \\
\hline 3 & 0.5625 \\
\hline 5 & 0.8789 \\
\hline 10 & 0.9961 \\
\hline 20 & 0.9999 \\
\hline 50 & 1 \\
\hline 100 & 1 \\
\hline
\end{tabular}

Hence the expected time delay also goes to 1 , as seen in the simulation results.

The next lowest expected time delay is for Scheme 1, followed by Scheme $2(q=1)$. Moreover, the expected time delay for Scheme $2(q=1)$ is substantially larger than that for the other two approaches. This is due to the fact that as compared to the other two approaches, that do not have an upper bound on the time horizon over which information is transferred between robots, in this case information about only a single (oldest) time step is communicated. Unless this information has been discarded (i.e., all robots have computed the centralized estimates for this time step), information about the next time step cannot be communicated. As a result, the expected time delay in obtaining the centralized estimates goes on increasing with time.

In order to compare the accuracy of the robot pose estimates generated using the proposed information-transfer schemes, we consider a team of $N=5$ robots moving in 2D with phase-shifted sinusoidal trajectories for 500 time steps (each time step has duration $0.05 \mathrm{sec}$ ). Each robot measures its linear, $v$, and rotational, $\omega$, velocity, as well as its distance, $d$, and bearing, $\theta$, to other robots in the team. The noise in these measurements is modeled as zeromean, white Gaussian and has standard deviation: $\sigma_{v}=$ $2 \% v, \sigma_{\omega}=1 \mathrm{deg} / \mathrm{sec}$ for the linear and rotational velocity measurements, respectively, and $\sigma_{d}=2 \% d$ and $\sigma_{\theta}=1 \mathrm{deg}$ for the corresponding distance and bearing measurements. Furthermore, as the robots move around, they randomly communicate with other robots in the team $\left(p^{j i}=p\right)$. When two robots communicate, the information transferred depends upon the chosen information-transfer scheme.

The CL algorithm is implemented by each robot using an EKF. When a robot calculates pose estimates at the current time step, if the measurements for that time step are unavailable for some robots in the team, the last available proprioceptive measurements from those robots are used for the propagation step of the EKF.

We employ the RMS error criterion to test the accuracy of these three approaches. Figs. 7 and 8 show the RMS error in the position and orientation estimates respectively, 


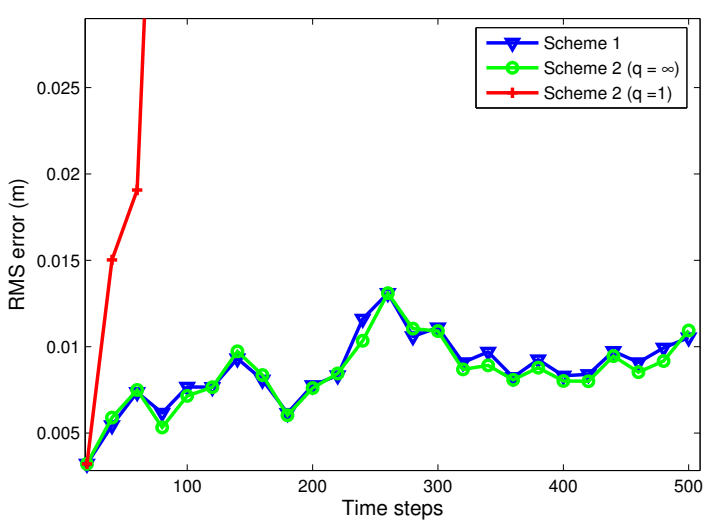

Fig. 7: RMS error in the robots' position estimates.

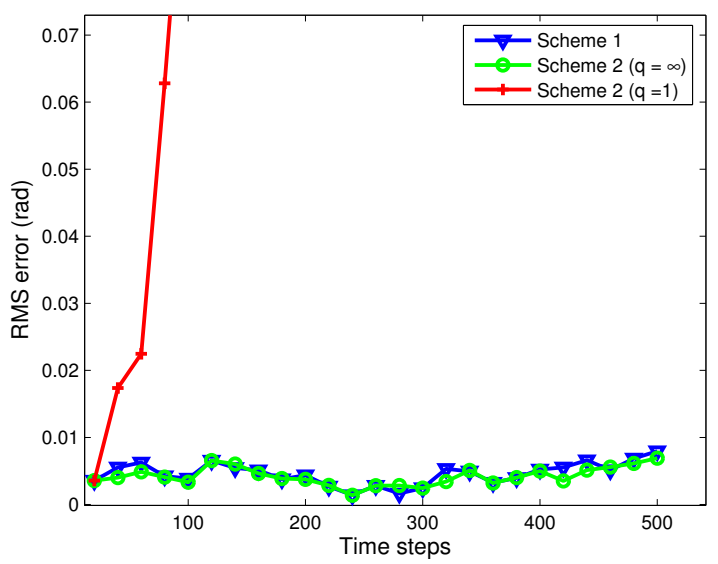

Fig. 8: RMS error in the robots' orientation estimates.

generated by robot 1 for the entire team. The RMS errors are plotted every 20 time steps for clarity. From the figures we see that for the chosen robot team of size $N=5$, the performances of Scheme $2(q=\infty)$ and Scheme 1 are almost indistinguishable from each other. Thus we can conclude that for small robot teams, using Scheme 1 instead of Scheme $2(q=\infty)$, will save valuable communication bandwidth per link without any significant loss of accuracy. But as the number of robots increases, Scheme 2 will outperform the other two schemes. This is due to the fact that even though all information necessary for generating the centralized estimates, i.e., $\hat{\mathbf{x}}_{k \mid k}^{k}$, may not be available to robot $i$ at time-step $k$, the set $T_{k}^{i}$ (i.e., locally-available information at time-step $k$ ) generated using Scheme $2(q=$ $\infty)$ is a superset of $T_{k}^{i+}$ generated using the other two schemes. Lastly, as expected, the pose estimates generated using Scheme $2(q=1)$ are the least accurate. Since the communication bandwidth available per link for scheme 2 $(q=1)$ is very restrictive, this translates into lower accuracy for the pose estimates. Thus depending upon the availability of communication resources and the accuracy requirements of the application, a suitable information-transfer scheme should be chosen.

\section{CONClusions And Future Work}

In this paper, we present a general framework for interrobot information-transfer schemes in multi-centralized $\mathrm{CL}$ under asynchronous communication. Depending upon the team's and the application's communication bandwidth availability, an appropriate information-transfer scheme can be selected to obtain pose estimates identical to the centralized estimates, but delayed. Furthermore, for these schemes we present detailed communication-complexity analysis, necessary and sufficient conditions to generate centralized estimates, and expected time delay analysis. Our future work will include the generalization of the analytical solution for the expected time delay using Scheme 2 to $N$ robots. On a broader level, an extension to this research is the development of time-varying information-transfer schemes that fully exploit the available communication resources at every time instance.

\section{REFERENCES}

[1] S. I. Roumeliotis, "Robust mobile robot localization: from single-robot uncertainties to multi-robot interdependencies," Ph.D. dissertation, Los Angeles, CA, 2000.

[2] R. Kurazume, S. Nagata, and S. Hirose, "Cooperative positioning with multiple robots," in Proc. of the IEEE International Conference on Robotics and Automation, San Diego, CA, May 8-13, 1994, pp. 12501257.

[3] I. M. Rekleitis, G. Dudek, and E. E. Milios, "Multi-robot collaboration for robust exploration," in Proc. of the IEEE International Conference on Robotics and Automation, San Francisco, CA, Apr. 24-28, 2000, pp. 3164-3169.

[4] A. Howard, M. J. Mataric, and G. S. Sukhatme, "Localization for mobile robot teams using maximum likelihood estimation," in Proc. of the IEEE/RSJ International Conference on Intelligent Robots and Systems, EPFL, Switzerland, Sep. 30-Oct. 5, 2002, pp. 434-439.

[5] N. Trawny, S. I. Roumeliotis, and G. B. Giannakis, "Cooperative multirobot localization under communication constraints," in Proc. of the IEEE International Conference on Robotics and Automation, Kobe, Japan, May 12-17, 2009, pp. 4394-4400.

[6] S. Panzieri, F. Pascucci, and R. Setola, "Multirobot localization using interlaced extended kalman filter," in Proc. of the IEEE/RSJ International Conference on Intelligent Robots and Systems, Beijing, China, Oct. 9-15, 2006, pp. 2816-2821.

[7] N. Karam, F. Chausse, R. Aufrere, and R. Chapuis, "Localization of a group of communicating vehicles by state exchange," in Proc. of the IEEE/RSJ International Conference on Intelligent Robots and Systems, Beijing, China, Oct. 9-15, 2006, pp. 519-524.

[8] A. Martinelli, "Improving the precision on multi robot localization by using a series of filters hierarchically distributed," in Proc. of the IEEE/RSJ International Conference on Intelligent Robots and Systems, San Diego, CA, Oct. 9-Nov. 2, 2007, pp. 1053-1058.

[9] A. Bahr, M. R. Walter, and J. J. Leonard, "Consistent cooperative localization," in Proc. of the IEEE International Conference on Robotics and Automation, Kobe, Japan, May 12-17, 2009, pp. 3415-3422.

[10] E. D. Nerurkar, S. I. Roumeliotis, and A. Martinelli, "Distributed maximum a posteriori estimation for multi-robot cooperative localization," in Proc. of the IEEE International Conference on Robotics and Automation, Kobe, Japan, May 12-17, 2009, pp. 1402-1409.

[11] K. Y. K. Leung, T. D. Barfoot, and H. Liu, "Decentralized localization of sparsely-communicating robot networks: A centralized-equivalent approach," IEEE Transactions on Robotics, vol. 26, no. 1, pp. 62-77.

[12] E. D. Nerurkar and S. I. Roumeliotis, "Multi-centralized cooperative localization under asynchronous communication," Dept. of Comp. Sci. \& Eng., University of Minnesota, Tech. Rep., 2010. [Online]. Available: http://wwwusers.cs.umn.edu/ nerurkar/Nerurkar_MultiCentralAsynchroComm.pdf 